\title{
Plasmonics in Atomically Thin Crystalline Silver Films
}

\author{
Zakaria M. Abd El-Fattah, ${ }^{\dagger, \ddagger}, \bigcirc$ Vahagn Mkhitaryan, ${ }^{\dagger, \bigcirc}$ Jens Brede, ${ }^{\S}{ }^{\circ}$ Laura Fernández, ${ }^{\|}$Cheng Li, ${ }^{\perp}$

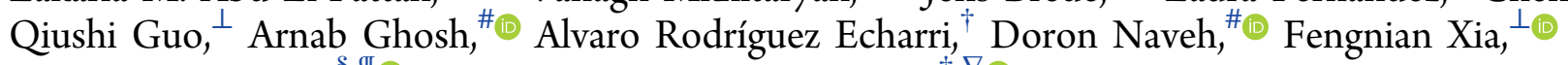 \\ J. Enrique Ortega, ${ }^{*}$, , Iङ and F. Javier García de Abajo ${ }^{*, \dagger}, \nabla_{\odot}$
}

${ }^{\dagger}$ ICFO-Institut de Ciencies Fotoniques, The Barcelona Institute of Science and Technology, 08860 Castelldefels, Barcelona, Spain ${ }^{\ddagger}$ Physics Department, Faculty of Science, Al-Azhar University, Nasr City, E-11884 Cairo, Egypt

${ }^{\S}$ Donostia International Physics Center, Paseo Manuel Lardizabal 4, 20018 Donostia, San Sebastián,Spain

"Centro de Física de Materiales CSIC-UPV/EHU and Materials Physics Center, 20018 San Sebastián, Spain

${ }^{\perp}$ Department of Electrical Engineering, Yale University, New Haven, Connecticut 06511, United States

${ }^{\#}$ Faculty of Engineering, Bar Ilan University, Ramat Gan 5290002, Israel

${ }^{\nabla}$ ICREA-Institució Catalana de Recerca i Estudis Avançats, Passeig Lluís Companys 23, 08010 Barcelona, Spain

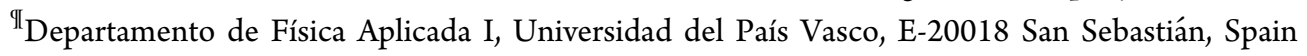

\section{Supporting Information}

ABSTRACT: Light-matter interaction at the atomic scale rules fundamental phenomena such as photoemission and lasing while enabling basic everyday technologies, including photovoltaics and optical communications. In this context, plasmons, the collective electron oscillations in conducting materials, are important because they allow the manipulation of optical fields at the nanoscale. The advent of graphene and other two-dimensional crystals has pushed plasmons down to genuinely atomic dimensions, displaying appealing properties such as a large electrical tunability. However, plasmons in these materials are either too broad or lying at low frequencies, well below the technologically relevant near-infrared regime. Here, we demonstrate sharp

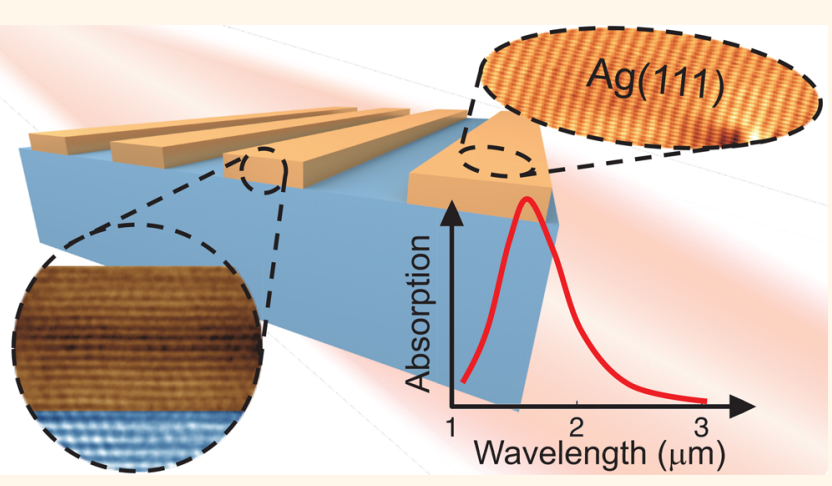
near-infrared plasmons in lithographically patterned wafer-scale atomically thin silver crystalline films. Our measured optical spectra reveal narrow plasmons (quality factor of $\sim 4$ ), further supported by a low sheet resistance comparable to bulk metal in few-atomic-layer silver films down to seven $\operatorname{Ag}(111)$ monolayers. Good crystal quality and plasmon narrowness are obtained despite the addition of a thin passivating dielectric, which renders our samples resilient to ambient conditions. The observation of spectrally sharp and strongly confined plasmons in atomically thin silver holds great potential for electro-optical modulation and optical sensing applications.

KEYWORDS: $2 D$ plasmonics, ultrathin plasmonics, $2 D$ materials, atomically thin silver, crystalline metal films

$\mathrm{T}$ he control of light at the nanoscale is a research frontier with applications in areas as diverse as biosensing, ${ }^{1,2}$ optoelectronics, ${ }^{3}$ nonlinear optics, ${ }^{4,5}$ quantum optics, ${ }^{6,7}$ and nanorobotics. ${ }^{8}$ Metallic nanostructures play a pivotal role in this context because they host collective electron oscillations, known as plasmons, which can interact strongly with light. This enables a large confinement of optical energy down to nanometer-sized regions, thereby enhancing the associated electromagnetic fields by several orders of magnitude relative to externally incident fields. ${ }^{9}$ Such appealing properties and the pursue of the noted applications have fueled intense research work into plasmonics to better understand and control these collective electronic excitations and cover a broad spectral range from the ultraviolet to the terahertz regimes. Progress has mainly relied on advances in nanofabrication and colloid chemistry, which allow the production of engineered metallic nanostructures with on-demand plasmonic response. $^{10,11}$

Plasmons in atomic-scale systems have emerged as a source of extraordinary properties resulting from the fact that they are

Received: February 28, 2019

Accepted: June 4, 2019

Published: June 4, 2019 

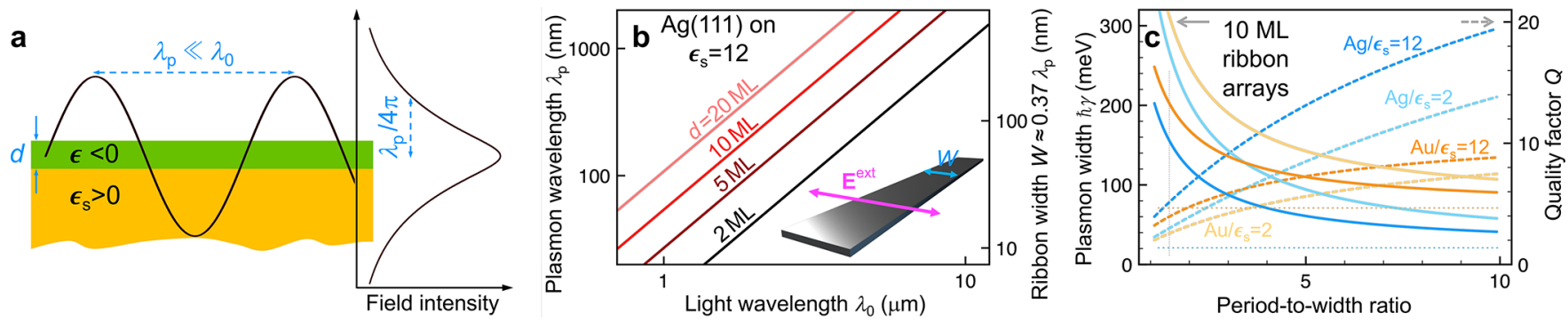

Figure 1. Properties of plasmons in atomically thin metal films. (a) The plasmon wavelength $\lambda_{\mathrm{p}}$ is small compared with the light wavelength $\lambda_{0}$, while the associated electric field extends a distance $\lambda_{\mathrm{p}} / 4 \pi$ away from the film (for $1 / e$ decay in field intensity), symmetrically on both sides of the interface (see the Methods section) regardless of dielectric environment and film composition. The sketch shows a cross section of an extended film (permittivity $\epsilon<0$ ) and substrate (permittivity $\epsilon_{s}>0$ ) in a plane perpendicular to the surface, along with the in-plane harmonic oscillation of the plasmon field (sine profile) and exponential out-of-plane decay of its intensity (right plot). (b) The plasmon wavelength scales linearly with metal thickness $d$ and quadratically with $\lambda_{0}$ as $\lambda_{\mathrm{p}}=d\left(\lambda_{0} / L_{1}\right)^{2}$, where $L_{1}$ depends on the choice of materials and is rather large $\left(L_{1} \approx 205 \mathrm{~nm}\right)$ for $\mathrm{Ag}$ on Si. A ribbon of width $W$ exhibits transverse dipolar resonances (i.e., with in-plane polarization across the ribbon) determined by $W \approx 0.37 \lambda_{\mathrm{p}}$. (c) In ribbon arrays, the plasmon width has a radiative component that scales linearly with both the metal thickness and the inverse of the period-to-width ratio (see Methods), and depends on the choice of metal and substrate permittivity (see labels), therefore affecting the quality factor $Q$ as shown here for $10 \mathrm{ML}$ metal at $\lambda_{0}=1.55 \mu \mathrm{m}$ wavelength. Dotted horizontal lines denote the long period limit for $\mathrm{Ag}$ and $\mathrm{Au}$. The dotted vertical line shows the ratio used in this work.

sustained by a comparatively small number of charge carriers. Electron energy-loss spectroscopy has been instrumental in revealing plasmons in systems such as $\mathrm{C}_{60}$ molecules, ${ }^{12}$ carbon and boron-nitride single-wall nanotubes, ${ }^{13,14}$ atomic gold wires grown on vicinal silicon surfaces, ${ }^{15}$ few-atomic-layer silver films, ${ }^{16}$ monolayer $\mathrm{DySi}_{2},{ }^{17}$ ultrathin indium ${ }^{18}$ and silicide ${ }^{19}$ wires, and graphene. ${ }^{20}$ Additionally, ultrathin TiN films have been demonstrated for refractory plasmonics, ${ }^{21,22}$ which contribute to configure the emerging field of transdimentional photonics. $^{23}$ Among these materials, high-quality graphene has been found to sustain low-energy plasmons when it is highly doped, exhibiting large electro-optical tunability, ${ }^{24,25}$ long lifetimes, ${ }^{26}$ and strong confinement compared with conventional plasmonic metals. ${ }^{27,28}$ Topological insulators ${ }^{29}$ and black phosphorus $^{30}$ have also been shown to display two-dimensional (2D) plasmons. Unfortunately, unlike noble-metal structures, the plasmons reported in these systems are either rather broad or lying at mid-infrared or lower frequencies, far from the technologically appealing near-infrared (NIR) regime. As a potential solution to this problem, electrochemically tunable plasmons have been revealed through optical spectroscopy in small polycyclic aromatic hydrocarbons, ${ }^{31,32}$ although their integration in fast commutation devices remains a challenge.

Atomically thin noble-metal films appear as a viable solution to achieve large electro-optical tunability ${ }^{33,34}$ within the NIR spectral range. However, crystalline quality is required to lower optical losses to the promised level for these materials in the plasmonic spectral region. Indeed, the presence of multiple facets in few-nanometer nanoparticles ${ }^{35,36}$ and sputtered films ${ }^{37}$ produce broad plasmons characterized by a quality factor $(Q=$ ratio of peak frequency to spectral width) of the order of $\sim 1$, which averts their use in cutting-edge plasmonic applications.

In this Article, we report on the fabrication and the excellent plasmonic and electrical properties of wafer-scale atomically thin crystalline silver films composed of only a few atomic layers. We use advanced surface-science techniques to fabricate and characterize $\operatorname{Ag}(111)$ films consisting of 7-20 atomic monolayers (MLs) on a clean $\mathrm{Si}(111)$ substrate, which we then cover with $\sim 1.5 \mathrm{~nm}$ of $\mathrm{Si}$ to passivate them from air. The high atomic quality of the samples, which we confirm through scanning tunneling microscopy (STM), angle-resolved photoelectron spectroscopy (ARPES), high-resolution transmission electron microscopy (HRTEM), and low-energy electron diffraction (LEED), allows us to resolve sharp electronic vertical quantum-well states (QWs) and measure very low sheet resistances for thin films down to $7 \mathrm{ML} \mathrm{Ag}(111)$ (1.65 $\mathrm{nm}$ thick, $\sim 20 \Omega / \mathrm{sq}$, just a factor of 2 higher than the bulk estimate). We obtain spectral evidence of confined plasmons by using electron-beam (e-beam) nanolithography to pattern ribbons on the silver films, resulting in measured plasmons with quality factors nearing $Q \approx 4$ for $10 \mathrm{ML}(\sim 2.4 \mathrm{~nm})$ films. These results reveal the ability of laterally patterned fewatomic-layer atomically flat silver to confine plasmons with similar lifetimes as bulk silver, thus extending $2 \mathrm{D}$ plasmonics into the technologically appealing NIR regime.

Like in graphene, ${ }^{38}$ metal films of small thickness $d$ in the few atomic-layer range allow us to dramatically reduce the inplane surface-plasmon wavelength $\lambda_{\mathrm{p}}$. In the Drude model (see the Methods section), we find $\lambda_{\mathrm{p}}$ to scale linearly with $d$ and quadratically with the light wavelength $\lambda_{0}$ as

$$
\lambda_{\mathrm{p}}=d \frac{\lambda_{0}^{2}}{L_{1}^{2}}
$$

(see Figure 1b), where $L_{1}$ is a characteristic length that depends on the combination of metal and substrate materials (e.g., $L_{1} \approx 205 \mathrm{~nm}$ for $\mathrm{Ag}$ on $\mathrm{Si}$ ). The confinement in the vertical direction is characterized by a symmetric exponential decay of the associated electric field intensity away from the film, extending a distance $\sim \lambda_{\mathrm{p}} / 4 \pi$ regardless of the choice of materials and metal thickness (Figure 1a). The comparatively small number of electrons that support the plasmons in atomically thin films makes them more susceptible to the environment, so that electrical gating with attainable carrier densities can produce significant plasmon shifts in single-atomlayer noble metals, ${ }^{33}$ while the addition and electrical gating of a graphene film results in dramatic modulation for thicker films up to a few nanometers. ${ }^{34}$ Likewise, the presence of an analyte can shift the plasmon resonance and introduce molecule spectral fingerprints enhanced by the near field of the plasmons, similar to what has been observed with graphene. ${ }^{2}$ However, besides such plasmon shifts, all of these applications 

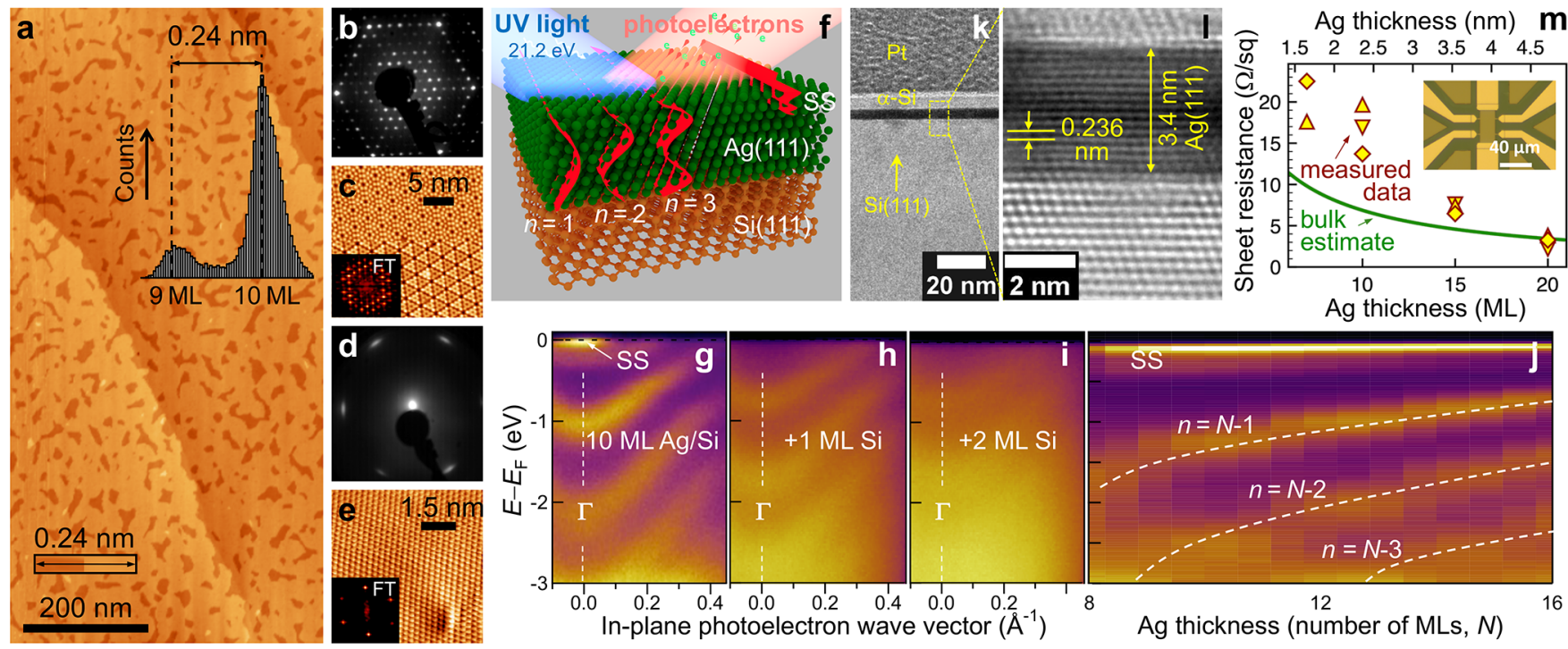

Figure 2. Fabrication and characterization of atomically thin crystalline silver films. (a) Scanning tunneling microscopy (STM) image of 10 ML $\operatorname{Ag}(111)$ on $\mathrm{Si}$. The histogram (upper inset) reveals the near-completion of the 10th layer (95\% area) with a small presence of $9 \mathrm{ML}$ (4\%, darker features) and $11 \mathrm{ML}(1 \%$, brighter features) islands (see color scale for out-of-plane distance). (b-d) Low-energy electron diffraction (LEED) of (b) the bare $\mathrm{Si}(111)(7 \times 7$ reconstruction) substrate and (d) after deposition of $10 \mathrm{ML} \mathrm{Ag}(111)$, along with atomic-scale STM details for both surfaces (panels $\mathrm{c}$ and e, respectively). Fourier transforms of the STM images are shown in the lower-left corners. (f) Sketch of $\operatorname{Ag}(111)$ film deposited on $\mathrm{Si}(111)$, along with its surface-state (SS) and the three lowest vertical quantum-well-state $(n=1-3)$ wave functions, here probed through angle-resolved photoemission spectroscopy (ARPES). ( $\mathrm{g}-\mathrm{i})$ ARPES intensity as a function of electron energy relative to the Fermi energy (vertical scale) and parallel wave vector for $(\mathrm{g})$ a $10 \mathrm{ML} \mathrm{Ag}(111) / \mathrm{Si}$ sample, and (h, $\mathrm{i})$ after coverage with 1 and 2 MLs of Si. (j) Evolution of the normal-emission ARPES intensity as a function of silver film thickness for 8-16 ML Ag(111) on $\mathrm{Si}$. Dashed curves are guides for the eye, corresponding the the top three states, with the state index $n$ (see $f$ ) varying with the number of layers $N$ as indicated by labels. ( $k, 1)$ High-resolution transmission electron microscopy (HRTEM) images of the transversal cross-section of a $14 \mathrm{ML} \mathrm{Ag}(111) / \mathrm{Si}$ sample, showing the silver atomic planes and their $0.236 \mathrm{~nm}$ separation. (m) Measured room-temperature sheet resistance for silver films consisting of $N=7-20 \mathrm{ML} \mathrm{Ag}(111) / \mathrm{Si}$ (symbols), compared with the $293{ }^{\circ} \mathrm{C}$ bulk estimate of $\sim(68.7 / \mathrm{N}) \Omega / \mathrm{sq}$ (solid curve). A total of three different devices have been measured for each value of $N$, and one of them is shown in the micrograph inset.

require spectrally narrow plasmons, so that spectral modulation results in strong changes in light transmission or scattering, and this, in turn, demands the fabrication of high-quality films.

\section{RESULTS AND DISCUSSION}

We epitaxially grow high-quality crystalline $\mathrm{Ag}(111)$ films on a $\mathrm{Si}(111)$-oriented wafer substrate ${ }^{39}$ with a controlled number of atomic monolayers under ultrahigh-vacuum (UHV) conditions (see the Methods section). Through fine tuning of the growth parameters, we achieve films consisting of a single crystal domain on a $\mathrm{cm}^{2}$ chip scale, as revealed by STM with atomic resolution (see Figures $2 \mathrm{a}$ and S2). The original $7 \times 7$ reconstruction of atomically flat $\mathrm{Si}$ (Figure 2c, where the upper and lower halves are empty and filled state images acquired with bias voltages of $+2 \mathrm{~V}$ and $-2 \mathrm{~V}$, respectively) is removed upon $\mathrm{Ag}$ deposition, leaving an atomically flat $\mathrm{Ag}$ surface (Figure 2e) that preserves crystal lattice orientation (cf. Figure $2 \mathrm{~b}, \mathrm{~d})$. We approach the targeted number of $\mathrm{Ag}(111)$ monolayers (10 ML in Figure $2 \mathrm{a}$ ) with just a $\sim 5 \%$ fraction of regions differing by $1 \mathrm{ML}$ thickness. We complete structural characterization by imaging a cross section of the film using HRTEM, which reveals a preservation of defect-free ordering of atomic $\operatorname{Ag}(111)$ monolayers (Figure 2k,l) on the Si crystal substrate.

Because plasmons are sustained by conduction electrons, we study the electronic band structure of the films, the small thickness of which produces discretization into a characteristic set of standing waves, encompassing vertical $\mathrm{QWs}^{40-42}$ (labeled by $n=1, \cdots$ in the sketch of Figure $2 \mathrm{f}$ ) and a surface-bound state (SS). Each of these QWs defines a band with nearly free parabolic dispersion (effective mass of $\sim 1$ ), as revealed by ARPES (Figure $2 \mathrm{~g}$ ), which also show narrow lineshapes. We passivate our films with $\mathrm{Si}$ (1.5 ML nominal thickness) to protect them during handling and patterning using e-beam nanolithography (see below). We note that highquality unpassivated $\operatorname{Ag}(111)$ films are stable during hours when brought from UHV to ambient conditions without patterning; ${ }^{43,44}$ however, strain in the $\mathrm{Ag}-\mathrm{Si}$ interface eventually leads to film dewetting (within days), initiated by pinholes $^{44}$ and leading to silver oxides and formation of rough films. The protective Si layer is rapidly oxidized upon exposure to air, while the underlying Ag film is unaffected for weeks (see Figure S1). We remark that the addition of the thin Si capping layer causes the SS to disappear but does not affect the QW states (Figure 2h,i). Control over thickness and high-quality of the films further allows us to experimentally observe a $\sim 1 / d$ scaling of the QW binding energies with increasing film thickness $d$ (Figure 2j), typical of a 1D particle-in-a-box system. We resolve QWs in all samples used in the present study, yielding an unambiguous determination of the number of layers in each Ag film. The presence and quality of the Ag film in the samples is further corroborated by ellipsometry measurements compared with bare $\mathrm{Si}$ substrates (see Figure S4).

It is a widely acknowledged fact that ultrathin metal films must experience strong surface scattering, and therefore, see their electrical resistance sharply increased, as previous studies have indicated. ${ }^{45-49}$ In contrast, the high crystal quality of our films produces very low levels of the sheet resistance (Figure 

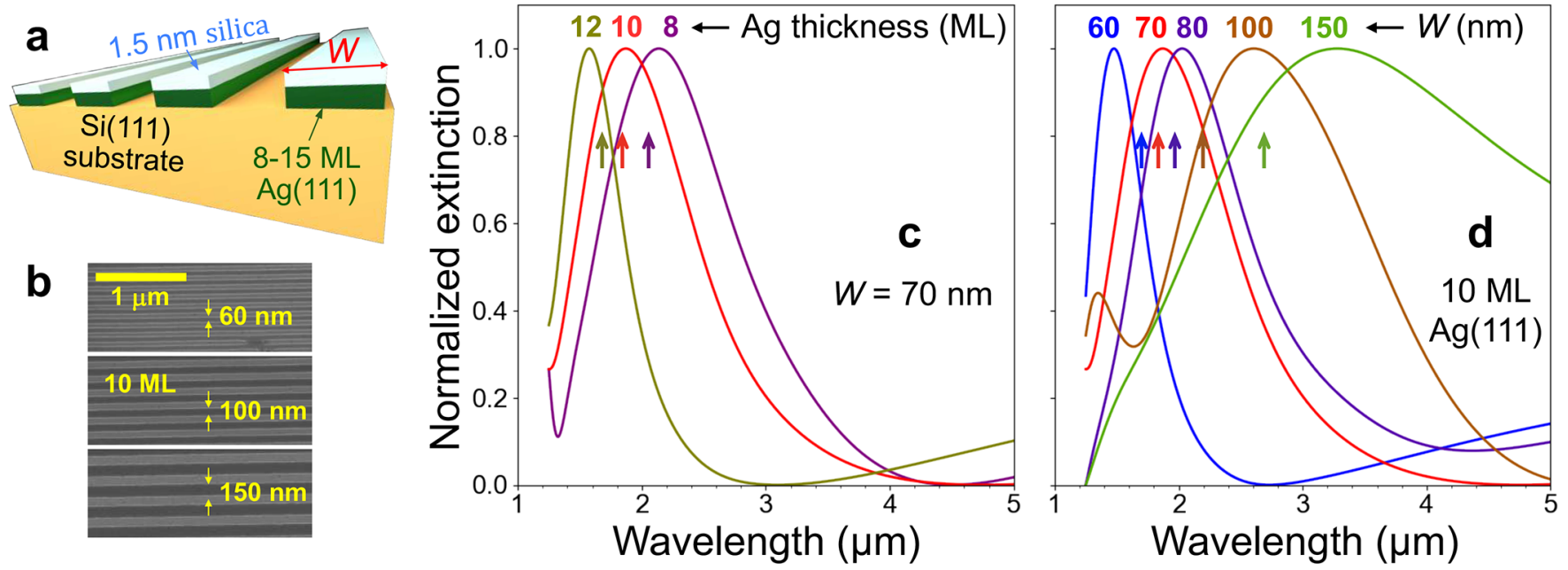

Figure 3. Plasmons in atomically thin crystalline silver nanoribbons. (a) Sketch of the ribbon arrays fabricated for this study. (b) Scanning electron microscopy (SEM) images of some of the structures in a $10 \mathrm{ML} \mathrm{Ag}(111) / \mathrm{Si}$ sample, with the targeted ribbon width (dark areas) indicated in each case. (c) Normalized optical extinction as experimentally measured for fixed ribbon width $W=70 \mathrm{~nm}$ and various metal film thicknesses (see labels). (d) The same as panel $\mathrm{c}$ for fixed metal thickness (10 ML) and varying ribbon width. Vertical arrows in panels $\mathrm{c}$ and $\mathrm{d}$ correspond to the analytical prediction of eq 2 for the color-coordinated plasmon wavelengths.
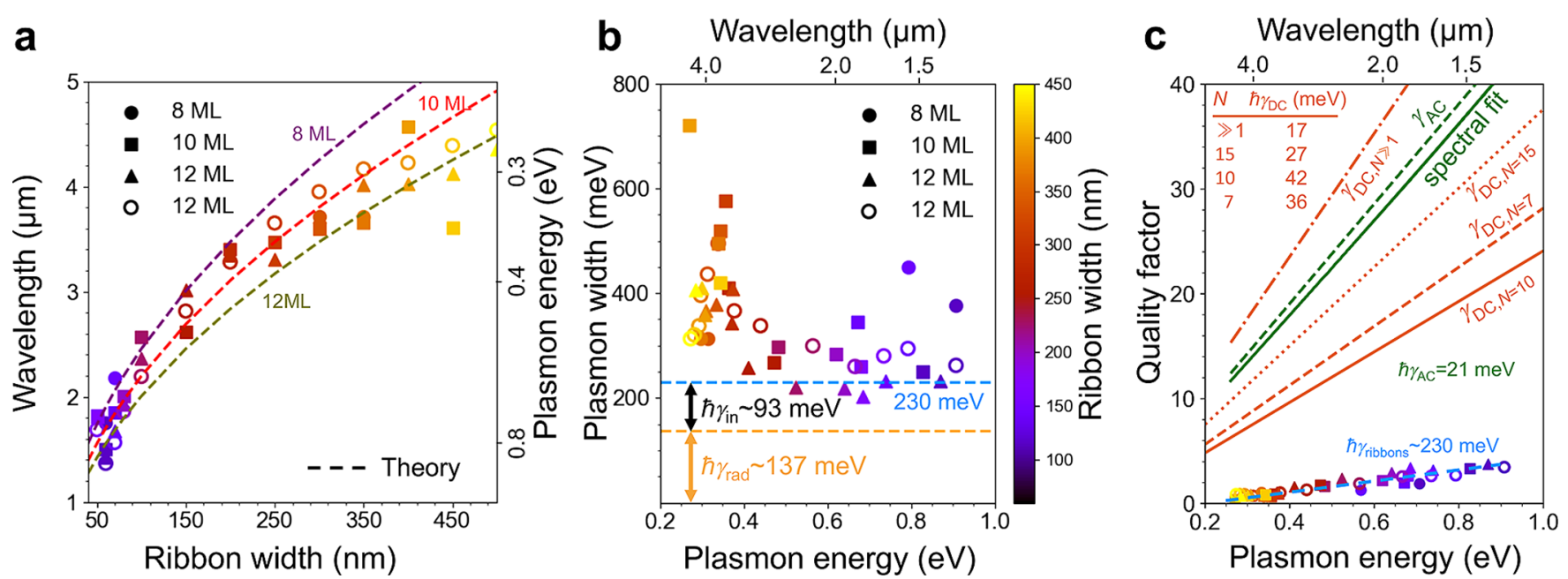

Figure 4. Engineering the wavelength and quality factor $Q$ of plasmons in atomically thin crystalline silver films. (a) Plasmon energy (right scale) and corresponding light wavelength (left scale) as a function of ribbon width. Experiment (symbols) is compared with simple analytical theory (dashed curves, eq 2). (b) Plasmon spectral width $\hbar \gamma$ as a function of plasmon energy $\hbar \omega$. Measured data from samples with various silver thicknesses are plotted using different symbols, with the ribbon width encoded in the color scale. The upper dashed horizontal line is a guide to the eye. The lower dashed line shows the radiative contribution to the damping $\hbar \gamma_{\text {rad }}$ according to eq 5 for 10 ML. (c) Quality factor (peak energy divided by fwhm spectral width, $Q=\omega / \gamma$ ) as predicted by the Drude model for silver films using different input values of the damping rate $\gamma$ (see the main text) compared with the experimental data taken from panel $b$.

$2 \mathrm{~m}$ ), as revealed by four-probe measurements (see Figure S3). In particular, we find the resistance to be only a factor of $\sim 2$ higher than the estimate based on the bulk resistivity of silver for films as thin as $7 \mathrm{ML} \mathrm{Ag}(111)$ (1.65 nm thickness). Because the film quality does not open new channels for inelastic collisions compared with the bulk, we attribute this factor of 2 to defects introduced by the capping Si layer, which has reduced crystallinity (see top of Figure 21 ), although some film damage during device fabrication cannot be ruled out. We thus attribute the large reduction of resistance in our films compared with previous studies, in which films had a polycrystalline morphology, to the high crystallinity and absence of grain boundaries obtained by our followed epitaxial procedure (see the Methods section). The present results thus establish a much lower bound for the role played by surface scattering in the electrical resistance of high-quality crystalline silver films.

Plasmons in atomically thin films are confined excitations with lateral wave vector $2 \pi / \lambda_{\text {p }}$ greatly exceeding the light wave vector $2 \pi / \lambda_{0}$, which prevents direct light-plasmon coupling. An additional source of lateral momentum is needed to break this optical momentum mismatch, such as that provided by a pattern in the films. In this work, we use e-beam nanolithography (see the Methods section) to carve ribbons with the desired range of widths $W \sim 50-500 \mathrm{~nm}$, which allow us to explore plasmon wavelengths $\lambda_{\mathrm{p}} \approx 2.7 \mathrm{~W}$ (Figure $1 \mathrm{~b}$ and the Methods section). The structure under consideration is sketched in Figure 3a, while scanning electron microscopy (SEM) images of some of the actual structures are shown in Figure $3 \mathrm{~b}$. The resulting measured optical spectra for different 
film thicknesses and ribbon widths are presented in Figure $3 \mathrm{c}, \mathrm{d}$, where plasmon redshifts are clearly observed when reducing the thickness or increasing the width, in qualitative agreement with the analytical formula

$$
\lambda_{0} \approx L_{1} \sqrt{2.7 W / d}
$$

(vertical arrows in Figure $3 \mathrm{c}, \mathrm{d}$ ), which predicts the light wavelength associated with the plasmon to scale linearly with the square root of the width-to-thickness aspect ratio $W / d$. This expression, which follows from the Drude model combined with the relation between $\lambda_{\mathrm{p}}$ and $W$ (see the Methods section), ${ }^{50}$ is in excellent agreement with a quantummechanical description of few-layer Ag films based upon the random-phase approximation combined with a realistic description of QWs in the films (see Figure S10). Analytically calculated spectra (see the Methods section) have a similar level of agreement with measurements and nearly coincide with full electromagnetic simulations (see Figures S8 and S9). Additionally, the spectra of Figure $3 \mathrm{c}, \mathrm{d}$ reveal an increase in plasmon broadening with increasing ribbon width (see below).

These plasmon characteristics are consistently corroborated upon inspection of different samples (see Figures S5 and S6), the wavelengths and spectral widths of which are summarized in Figure 4. In particular, when plotting the observed plasmon wavelength as a function of ribbon width, we obtain a good agreement with eq 2 despite deviations in individual structures, which we attribute to a variability in the actual width of the patterned ribbons. Additionally, we find a roughly constant plasmon width $\hbar \gamma \approx 230 \mathrm{meV}$, which gives rise to a linear increase in $Q=\omega / \gamma$ with plasmon energy $\hbar \omega$ (see Figure $4 b, c$ and the quality-factor extraction procedure in Figure S7). In our experiments, we find values of $Q$ approaching 4 at plasmon energies near $1 \mathrm{eV}$. These spectrally narrow plasmons are made possible by the small thickness of our metal films combined with their crystalline quality. Indeed, polycrystalline films in the few nanometer range hardly reach $Q \approx 1 .^{37}$ Additionally, spatially confined NIR plasmons in noble metals require the use of high aspect ratios $W / d \approx 20$; before the present study, high-quality structures could only be obtained for much thicker films, therefore involving larger $W$, and in consequence producing substantially broader plasmons due to coupling to radiation. Likewise, NIR plasmons in metallic colloids demand large particle aspect ratios, for which the observed quality factors are significantly smaller than 4 due to radiative losses as well, ${ }^{51}$ while in contrast to our films, the strategy of bringing the particle size to the few-nanometer range introduces additional plasmon quenching originating in finite-size effects ${ }^{36}$ and thereby limiting the achievable $Q$.

The plasmon quality factors in our ribbon arrays are partially limited by radiative losses (see Figure 1c). Indeed, as shown in the Methods section, the total plasmon damping rate $\gamma=\gamma_{\text {in }}+$ $\gamma_{\mathrm{rad}}$ is the sum of an intrinsic component $\gamma_{\text {in }}$ and a geometrydependent radiative component $\gamma_{\mathrm{rad}}=\Gamma \times W d / a$, where $\hbar \Gamma \approx$ $88 \mathrm{meV} / \mathrm{nm}$ for $\operatorname{Ag}(111)$ films on silicon. For our experiments, we fabricate ribbons with a period-to-width ratio $a / W=1.5$, which yields $\hbar \gamma_{\mathrm{rad}} \approx 137 \mathrm{meV}$ for $10 \mathrm{ML}$ films. This value is shown in Figure $4 \mathrm{~b}$ as a lower dashed line; so we are left with an intrinsic damping $\hbar \gamma_{\text {in }} \approx 93 \mathrm{meV}$, which is still over 4 times larger than the bulk value of $21 \mathrm{meV}$ derived from the measured permittivity of bulk silver. ${ }^{52}$ We note that radiative losses should be negligible for arrays of large period-to-width ratio, thus suggesting a direct way to improve the quality factor with the same film quality (see Figure S8).
The excess of intrinsic damping is presumably originating in sample damage incurred during the etching processes used for e-beam nanolithography (see the Methods section). Now, the question arises, how high can $Q$ be for confined plasmons based upon high-quality metal films consisting of a few atomic layers (e.g., < $2 \mathrm{~nm}$ for $8 \mathrm{ML} \mathrm{Ag}(111)$ )? We address this question by comparing our measured $Q$ 's with different estimates based upon the Drude expression $Q=\omega / \gamma_{\text {in }}$ neglecting radiative losses (Figure $4 \mathrm{c}$ ). Assuming the value $\hbar \gamma_{\mathrm{AC}}=21 \mathrm{meV}$ obtained by fitting the measured $\mathrm{Ag}$ permittivity $^{52}$ to a Drude tail in the $<1 \mathrm{eV}$ spectral region, we find $Q>40$ (an order of magnitude larger than those observed here), in agreement with predictions based on the estimate of $Q$ given by $-\operatorname{Im}\{\epsilon\} / \operatorname{Re}\{\epsilon\} .{ }^{53}$ This is also in good agreement with both the quality factors of spectra calculated in the long-wavelength limit (spectral fit) and the estimate obtained form the measured bulk DC conductivity $\left(\hbar \gamma_{\mathrm{DC}, N \gg 1}\right.$ $\approx 17 \mathrm{meV}$ ). We note that the sheet resistance measured from our films (Figure $2 \mathrm{~m}$ ) leads according to the Drude model to predicted values $Q>20$ for $10 \mathrm{ML} \operatorname{Ag}(111)$ films (see the Methods section) in the absence of radiative losses.

\section{CONCLUSIONS}

In brief, we report well-defined plasmons in atomically flat $\operatorname{Ag}(111)$ films grown on $\mathrm{Si}(111)$, with thickness as low as 8 $\mathrm{ML}(\sim 1.9 \mathrm{~nm})$. The measured quality factors reach values of $\sim 4$. Further improvement of these results should include the exploration of thinner films down to 1-3 ML, which are, however, challenging because of the strain associated with the $\mathrm{Ag}-\mathrm{Si}(111)$ interface. Following a two-step process (i.e., deposition at low temperature of $\sim 100 \mathrm{~K}$ followed by annealing to $300 \mathrm{~K}$ ), we find the lowest thickness needed to produce atomically flat films using this procedure to be $6 \mathrm{ML}$. Nevertheless, $2 \mathrm{ML} \mathrm{Ag}(111)$ films have been recently reported by employing a $\mathrm{Ga} / \mathrm{Si}$ buffer layer, ${ }^{54}$ showing rather flat surfaces and well-defined quantum-well states. ${ }^{55}$ In our films, the crystalline quality of the fabricated $\operatorname{Ag}(111)$ films, which exhibit a clean electronic band structure consisting of quantized QWs, combined with the crystalline quality of the substrate, permit ruling out inelastic electron and plasmon scattering due to imperfections. However, the noncrystalline protecting capping layer can introduce inelastic coupling channels. Additionally, the etching processes used during ebeam lithography can cause sample damage, to which we attribute the reduction by half in film electrical conductance and by a factor of $\sim 5$ in optical quality factor with respect to the maximum estimate in the studied spectral range, while another factor of $>2$ in quality factor can be gained by reducing radiative losses (e.g., by increasing the period-to-width ratio of the ribbon arrays). Further improvement in nanofabrication could therefore increase the achieved plasmon quality factors. Nevertheless, the plasmons here observed should be already sufficiently narrow to produce large electro-optical modulation in the $\mathrm{NIR}^{34}$ while their reduced vertical and lateral size (down to $\sim 20$ and $\sim 50 \mathrm{~nm}$ at $0.8 \mathrm{eV}$, see Figures $1 \mathrm{a}$ and $4 \mathrm{a}$ ) are ideally suited for enhancing the interaction with neighboring molecules, thus holding great potential for optical sensing.

\section{METHODS}

Fabrication of Atomically-Thin Silver Films. Our $\mathrm{Ag} / \mathrm{Si}(111)$ samples were prepared inside an UHV chamber at $1.0 \times 10^{-10} \mathrm{mbar}$ base pressure. We used $4 \mathrm{~mm} \times 12 \mathrm{~mm} \mathrm{n}$-doped $\mathrm{Si}(111)$ chips with 
specific resistance $120-340 \Omega \mathrm{cm}$ as bare substrates. The dopant concentration of $\mathrm{Si}\left(1.3-3.7 \times 10^{13} \mathrm{~cm}^{-3}\right)$ was chosen to guarantee the electrical conduction required by surface science techniques while not influencing the plasmonic performance of the silver films. Once inside the UHV chamber, the $\mathrm{Si}(111)$ chips were degassed overnight at $900 \mathrm{~K}$ and subsequently flashed to $1400 \mathrm{~K}$ for $20-30 \mathrm{~s}$ to remove the native silicon oxide. The sample temperature was slowly reduced to $600 \mathrm{~K}$, maintained at this temperature for $30 \mathrm{~min}$, and then cooled to room temperature. This resulted in the formation of a defect-free, atomically clean $\mathrm{Si}(111)$ surface with a $7 \times 7$ reconstruction. Silver atoms were sublimated from an electron-bombardment evaporator, which was calibrated to submonolayer accuracy using a quartz microbalance monitor in combination with probing of the distinct 1$2 \mathrm{ML} \mathrm{Ag} / \mathrm{Cu}(111)$ surface states by photoemission. ${ }^{56}$ Silver films were grown on $\mathrm{Si}(111)$ following this two-step process. The $\mathrm{Si}(111)$ substrate was kept at 100-120 K during Ag deposition and slowly annealed to room temperature afterward. ${ }^{57}$ The deposition rate was $\sim 0.3 \mathrm{ML} / \mathrm{min}$, although a similar film quality was obtained within the $0.1-0.5 \mathrm{ML} / \mathrm{min}$ range; the crucial parameter here is the deposition temperature, which was required to be $\sim 100 \mathrm{~K}$.

Surface-Science Characterization. The atomic and electronic structure of the $\mathrm{Si}$ substrate and the grown $\mathrm{Ag}$ films were characterized by LEED, STM, and ARPES. STM data were collected using an Omicron VT setup operating at room temperature. ARPES measurements were performed using a SPECS Phoibos 150 electron analyzer equipped with a monochromatized $\mathrm{He}$ gas discharge lamp operating at the $\mathrm{He} \mathrm{I} \alpha$ excitation energy $(21.2 \mathrm{eV})$, with an electron energy and angular resolution of $30 \mathrm{meV}$ and $0.1^{\circ}$, respectively. The diameter of the UV light beam was $\sim 0.5 \mathrm{~mm}$ at the sample surface. Sample transfer between STM and ARPES setups was made without breaking UHV conditions. Prior to atmosphere exposure, the samples were capped by a $\mathrm{Si}$ protection layer ( $1.5 \mathrm{~nm}$ nominal thickness), evaporated by direct heating of a Si chip with the same doping level as the substrate. The robustness and aging of the films was monitored by X-ray photoemission spectroscopy (XPS; see Figure S1).

HRTEM Characterization. Electron-transparent $(<50 \mathrm{~nm}$ thickness) cross-sectional lamellas of the samples were prepared by first sputtering a platinum layer for protection, followed by carving using a FEI Helios NanoLab 600 dual beam SEM/focused-ion-beam (FIB) system. After the transfer of the lamellas to a copper grid, they were imaged using a JEOL JEM-2100 high-resolution transmission electron microscope operated at $200 \mathrm{kV}$.

Sheet Resistance Measurements. Ultrathin silver films were etched into a Hall-bar structure by argon plasma using an Oxford Plasmalab 100 reaction-ion etching (RIE) system. A poly(methyl methacrylate) (PMMA) layer was used as the etch mask. Contact electrodes were formed by depositing a $\mathrm{Cr} / \mathrm{Au} / \mathrm{Al}(3 / 60 / 190 \mathrm{~nm})$ layer followed by lift-off. All structures were patterned by a Raith EBPG 5000+ electron-beam lithography system. A four-probe scheme $^{58}$ (Figure S3) was used to extract the sheet resistance. The electrical characterization was performed in a Lakeshore probe station operating at $7 \times 10^{-5} \mathrm{mbar}$. An Agilent B1500A semiconductor parameter analyzer was used for all electrical measurements.

Electron-Beam Nanolithography. Passivated silver-film chips were uniformly spin-coated with $\sim 100 \mathrm{~nm}$ ZEP520A resist for $1 \mathrm{~min}$ at $6000 \mathrm{rpm}$. Ribbons were then written using a RAITH150-Two electron-beam lithography system followed by development in amyl acetate and reactive-ion etching for $\sim 1$ min with an $\mathrm{Ar}$ and $\mathrm{CHF}_{3}$ mixture in a RIE Oxford Plasmalab 80 Plus system. Periodic arrays of 50-1000 nm wide ribbons were fabricated with a footprint of $200 \mu \mathrm{m}$ $\times 200 \mu \mathrm{m}$ per sample and a $\sim 1.5$ period-to-width ratio. Importantly, although standard procedures usually involve baking at $150-180^{\circ} \mathrm{C}$ after spin-coating to induced a phase transition to glass in the resist, we skipped this step to avoid Ag film damage, at the expense of having a more fragile resist that required careful calibration of the RIE gas mixture and etching time to preserve the etching mask.

Optical Characterization. We used a SOPRA GES-5E system to perform ellipsometry (Figure S4) for incidence angles in the $60-75^{\circ}$ range over the UV-NIR photon energy region $(1.5-5 \mathrm{eV})$. Optical transmission/reflectance spectra (Figures S5 and S6) were collected using a Bruker Hyperion Fourier-transform infrared (FTIR) spectrometer operating in the $1.3-17 \mu \mathrm{m}$ range.

Analytical Simulations. The plasmon dispersion relation (parallel wave vector $k_{\|}$as a function of frequency $\omega$ ) of a homogeneous thin film is given in the quasistatic limit by ${ }^{38}$

$$
k_{\|}=\frac{i \omega\left(\epsilon_{1}+\epsilon_{2}\right)}{4 \pi \sigma}
$$

where $\epsilon_{1}$ and $\epsilon_{2}$ are the permittivities of the media on either side of the film, while $\sigma$ is the $2 \mathrm{D}$ conductivity. Assuming local response, we write the latter as

$$
\sigma=(\mathrm{i} \omega / 4 \pi)(1-\epsilon) d
$$

which is proportional to the film thickness $d$ and where $\epsilon$ stands for the metal permittivity; this is an excellent approximation for the materials and film thicknesses under consideration even when compared with quantum-mechanical simulations (see Figure S10). Adopting the Drude model, ${ }^{59}$ we approximate $\epsilon \approx 1-\omega_{\text {bulk }}^{2} / \omega(\omega+$ $\left.\mathrm{i} \gamma_{\text {in }}\right)$ in terms of the bulk plasma frequency $\omega_{\text {bulk }}$ and the intrinsic damping rate $\gamma_{\text {in }}$ (assuming $\omega \ll \omega_{\text {bulk }}$ ), which leads to the dispersion relation $k_{\|} d \approx\left(\epsilon_{1}+\epsilon_{2}\right) \omega\left(\omega+\mathrm{i} \gamma_{\text {in }}\right) / \omega_{\text {bulk }}^{2}$ and this in turn allows us to write the in-plane plasmon wavelength defined by $\lambda_{\mathrm{p}}=2 \pi / \operatorname{Re}\left\{k_{\|}\right\}$as $\lambda_{\mathrm{p}}=d\left(\lambda_{0} / L_{1}\right)^{2}$ (i.e., eq 1 in the main text), where

$$
L_{1}=\sqrt{2 \pi\left(\epsilon_{1}+\epsilon_{2}\right)} \frac{c}{\omega_{\text {bulk }}}
$$

and $\lambda_{0}$ is the free-space light wavelength. For Ag films $\left(\hbar \omega_{\text {bulk }} \approx 9.17\right.$ $\left.\mathrm{eV}^{52}\right)$ deposited on silicon $\left(\epsilon_{1} \approx 12\right)$ and coated with ZEP502A resist $\left(\epsilon_{2} \approx 2.4\right)$, we find $L_{1} \approx 205 \mathrm{~nm}$, which renders $\lambda_{\mathrm{p}} \ll \lambda_{0}$ at light wavelengths below $\sim 5 \mu \mathrm{m}$ when $d$ spans a few atomic layers (below $\sim 15 \mathrm{ML}$ ), thereby justifying our using the quasistatic limit, although retardation effects can become apparent for longer wavelengths and thicker films. Incidentally, the resist is not removed from the samples before plasmon measurements, but the penetration depth $\lambda_{\mathrm{p}} / 4 \pi$ is smaller than the resist thickness $(\sim 100 \mathrm{~nm})$, thus justifying the use of the resist permittivity in the above expression for $L_{1}$.

We remark that the above results assume a small film thickness $d$ compared with the plasmon wavelength $\lambda_{\mathrm{p}}$, while the reduction of the metal film response to a surface conductivity is valid if $d$ is also small compared with the skin depth $\lambda_{0} /(2 \pi \operatorname{Im}\{\sqrt{\epsilon}\}) \approx c / \omega_{\text {bulk }} \sim 20 \mathrm{~nm}$ in Ag. Additionally, in the quasistatic limit, the electric field $\mathbf{E}$ is longitudinal $(\nabla \times \mathbf{E}=0)$ and divergenceless $(\nabla \cdot \mathbf{E}=0)$, therefore displaying a symmetric pattern relative to the negligibly thick film (we refer to a recent study ${ }^{60}$ for more details). In particular, the electric field associated with the plasmon has symmetric (antisymmetric) inplane (out-of-plane) components with respect to the normal coordinate $z$ and admits the expression ${ }^{60} \propto[\hat{\mathbf{x}}+\mathrm{i} \operatorname{sign}(z) \hat{\mathbf{z}}] \mathrm{e}^{k_{\|}(\mathrm{i} x-|z|)}$ for propagation along the in-plane direction $x$, from which an exponential decay away from the film is predicted with a $1 / e$ fall in intensity at a distance $\lambda_{\mathrm{p}} / 4 \pi$ from the film (see Figure 1a in the main text). We note that the field is however asymmetric if the film thickness is not small compared with both the plasmon wavelength and the metal skin depth. The above expression for the field also allows us to write the in-plane plasmon propagation distance (for $1 / e$ decay in intensity) as $L_{\mathrm{p}}=1 / 2 \operatorname{Im}\left\{k_{\|}\right\}$. Using the dispersion relation noted above, we find $L_{\mathrm{p}}=\lambda_{\mathrm{p}} L_{2} / \lambda_{0}$, where $L_{2}=c / 2 \gamma_{\text {in }}$ (e.g., taking $\hbar \gamma_{\text {in }}$ $=21 \mathrm{meV}$ for $\mathrm{Ag}$, as obtained from optical data, ${ }^{52}$ we have $L_{2}=4.7$ $\mu \mathrm{m}$ ); the propagation distance is then $L_{2} / \lambda_{0}$ (independent of metal thickness) times the plasmon wavelength (proportional to metal thickness). Incidentally, a plasmon lifetime $1 / \gamma_{\text {in }}$ is directly inherited from the Drude model in the absence of radiative losses (a good approximation for $\lambda_{\mathrm{p}} \ll \lambda_{0}$ ) and substrate absorption (Si losses are negligible in the studied spectral range within the $\lambda_{\mathrm{p}} / 4 \pi$ plasmon penetration depth), leading to a plasmon quality factor (frequency-towidth ratio) $Q=\omega / \gamma_{\text {in }}$. This relation is used in Figure 4 c of the main text with various estimates for $\gamma_{\text {in }}$ (see below as well). We also find useful to write the propagation distance as $L_{\mathrm{p}}=\lambda_{\mathrm{p}} \mathrm{Q} / 4 \pi$.

For ribbon arrays, plasmons are excited under transverse polarization (i.e., with the electric field oriented across the width of the 
ribbons, see Figure $1 \mathrm{~b}$ in the main text), whereas a featureless weak absorption is produced when the incident light field is parallel to the ribbons. Consequently, we concentrate on the former in what follows and adopt a previously reported $\operatorname{model}^{38}$ to calculate the normalincidence transverse-polarization transmission coefficient as

$$
t=\frac{1}{\bar{n}}\left[1+\frac{\mathrm{i} S}{\widetilde{\alpha}^{-1}-G}\right]
$$

where $\bar{n}=\left(1+\sqrt{\epsilon_{\mathrm{Si}}}\right) / 2$ is the average refractive index of the media above (air, neglecting the resist layer in the coupling to radiation) and below $(\mathrm{Si})$ the metal layer, $\tilde{\alpha}$ is the ribbon polarizability per unit length, $S=4 \pi^{2} / a \lambda_{0} \bar{n}$ describes radiative coupling, $a$ is the lattice period, $G=2 \pi^{2} / 3 a^{2} \bar{\epsilon}+\mathrm{i} S$ accounts for inter-ribbon interactions in the dipolar approximation, and $\bar{\epsilon}=\left(1+\epsilon_{\mathrm{Si}}\right) / 2$ is the average permittivity of the surrounding media. We express the polarizability as

$$
\widetilde{\alpha} \approx-W^{2} \bar{\epsilon} \zeta_{1}^{2} \frac{1}{1 / \eta_{1}+\mathrm{i} \omega W \bar{\epsilon} / \sigma}
$$

in terms of the $2 \mathrm{D}$ conductivity of the metal $\sigma$ and only consider the dominant contribution of the dipolar plasmon resonance corresponding to parameters ${ }^{61} \eta_{1} \approx-0.0921+0.0233 \mathrm{e}^{-8.9 d / W}$ and $\zeta_{1} \approx 0.959-$ $0.016 \mathrm{e}^{-39 d / W}$, which depend on the ribbon thickness-to-width aspect ratio $d / W$. Finally, the $2 \mathrm{D}$ conductivity is related to the metal permittivity as $\sigma=(\mathrm{i} \omega / 4 \pi)\left[\left(1-\epsilon_{\mathrm{Ag}}\right) d+\left(1-\epsilon_{\mathrm{c}}\right) d_{\mathrm{c}}\right]$, where we approximate the capping layer of thickness $d_{\mathrm{c}}=1.5 \mathrm{~nm}$ as an additional term in $\sigma$ with $\epsilon_{\mathrm{c}}=2$. We use tabulated optical data for silver $^{52}\left(\epsilon_{\mathrm{Ag}}\right)$ and crystalline silicon ${ }^{62}\left(\epsilon_{\mathrm{Si}}\right)$. Reassuringly, the analytical theory just presented produces spectra in nearly full agreement with numerical electromagnetic simulations (see Figures S8 and S9). Incidentally, this analysis of ribbon arrays ignores the resist, which our numerical simulations (not shown) predict to only cause minor plasmon redshifts.

The transverse dipolar plasmon of a single ribbon is signaled by a divergence in $\tilde{\alpha}$ (i.e., $\mathrm{i} \omega \bar{\epsilon} / \sigma=-1 / \eta_{1} W$ ), which combined with the dispersion relation of the extended film $k_{\|}=\mathrm{i} \omega \bar{\epsilon} / 2 \pi \sigma \approx 2 \pi / \lambda_{\mathrm{p}}$ leads to the condition

$$
W=\frac{\lambda_{\mathrm{p}}}{4 \pi^{2}\left(-\eta_{1}\right)} \approx 0.37 \lambda_{\mathrm{p}}
$$

for $d \ll W$. Adopting this expression and neglecting inter-ribbon interactions, we can use eq 1 to readily obtain eq 2 in the main text. It should be noted that inter-ribbon interaction can produce a small redshift correction in the plasmon position (see Figure S8).

We find it convenient to arrange the above above expressions by neglecting the capping layer and approximating the silver permittivity as $\epsilon_{\mathrm{Ag}} \approx 1-\omega_{\text {bulk }}^{2} / \omega\left(\omega+\mathrm{i} \gamma_{\mathrm{in}}\right)$ to express the transmission coefficient of the array (eq 3 ) as

$$
t=\frac{1}{\bar{n}}\left[1+\frac{\mathrm{i} \omega \gamma_{\mathrm{rad}}}{\omega_{\mathrm{p}}^{2}-\omega(\omega+\mathrm{i} \gamma)}\right]
$$

where

$$
\omega_{\mathrm{p}}=\omega_{\text {bulk }} \sqrt{\frac{1}{4 \pi \bar{\epsilon}\left(-\eta_{1}\right)} \frac{d}{W}-\frac{\pi \zeta_{1}^{2}}{6 \bar{\epsilon}} \frac{W d}{a^{2}}}
$$

is the resulting plasmon resonance of the array under normal incidence, whereas

$$
\gamma=\gamma_{\text {in }}+\gamma_{\text {rad }}
$$

is the total plasmon damping rate, contributed by the intrinsic component $\gamma_{\text {in }}$ and a radiative component

$$
\gamma_{\text {rad }}=\frac{\zeta_{1}^{2}}{2 \bar{n}} \frac{\omega_{\text {bulk }}^{2}}{c} \frac{W d}{a}
$$

The first term inside the square root of eq 4 describes the plasmon frequency of the isolated ribbon, while the second term accounts for a redshift due to inter-ribbon interaction. We note that radiative damping (eq 5) decreases with increasing array period $a$, so sharper plasmons are expected in the limit of large separations, for which $\gamma \approx$ $\gamma_{\text {in }}$ (see Figure 1c); incidentally, we have neglected radiative contributions to the damping of individual ribbons under the assumption $W \ll \lambda_{0}$. When we specify eq 5 to $\operatorname{Ag}(111)$ ribbons on silicon, we find $\gamma_{\mathrm{rad}}=\Gamma \times W d / a$, where $\hbar \Gamma=\zeta_{1}^{2} \hbar \omega_{\text {bulk }}^{2} /(2 \bar{n} c) \approx 88$ $\mathrm{meV} / \mathrm{nm}$.

Drude Damping Estimated from the Electrical Resistance. We use the expression

$$
\rho_{0, \mathrm{CGS}}[\mathrm{s}] \approx\left(4 \pi \times 8.854 \times 10^{-12}\right) \times \rho_{0, \mathrm{SI}}[\Omega \mathrm{m}]
$$

to convert DC resistivities from SI to CGS units. Then, we use the Drude model to write the damping rate as

$$
\gamma_{\text {in }}=(4 \pi)^{-1} \omega_{\text {bulk }}^{2} \rho_{0, \mathrm{CGS}}
$$

Damping rates in Figure $4 \mathrm{c}$ are obtained by applying these formulas to the SI resistivities $\rho_{0, S I}=1.62 \times 10^{-8} \Omega \mathrm{m}$ for bulk silver $\left(\gamma_{\mathrm{DC}, N \gg 1}\right)$ and $\rho_{\mathrm{S}} N d_{111}$ for silver films consisting of $N \mathrm{Ag}(111)$ atomic layers $\left(\gamma_{\mathrm{DC}, N}\right)$, where $d_{111}=0.236 \mathrm{~nm}$ is the atomic layer spacing and $\rho_{\mathrm{S}}$ is the average sheet resistance (for each value of $N$ ) obtained from the data points presented in Figure $2 \mathrm{~m}$.

\section{ASSOCIATED CONTENT}

\section{S Supporting Information}

The Supporting Information is available free of charge on the ACS Publications website at DOI: 10.1021/acsnano.9b01651.

Additional figures showing the surface quality upon $\mathrm{Si}$ capping and after exposure to ambient conditions, examples of characteristic surface thickness distributions, a micrograph of a sheet resistance measurement device, ellipsometry measurements, measured transmission spectra, a description of the method used to extract the plasmon quality factor from the measured spectra, a theoretical study of the effect of ribbon array spacing, a comparison between quantum-mechanical and classical theoretical descriptions of plasmons in thin metal films, and a comparison of measured spectra with analytical and numerical simulations (PDF)

\section{AUTHOR INFORMATION}

\section{Corresponding Authors}

*E-mail: enrique.ortega@ehu.es.

*E-mail: javier.garciadeabajo@nanophotonics.es.

ORCID

Jens Brede: 0000-0002-4946-8160

Arnab Ghosh: 0000-0003-1828-9837

Doron Naveh: 0000-0003-1091-5661

Fengnian Xia: 0000-0001-5176-368X

J. Enrique Ortega: 0000-0002-6643-806X

F. Javier García de Abajo: 0000-0002-4970-4565

\section{Author Contributions}

OZ.M.A. and V.M. contributed equally to this work.

\section{Notes}

The authors declare no competing financial interest.

\section{ACKNOWLEDGMENTS}

We thank Marta Autore, Josep Canet-Ferrer, Rainer Hillenbrand, Johan Osmond, and Frederik Schiller for technical support and helpful discussions. V.M. and F.J.G.A. gratefully acknowledge generous help and hospitality from Luis Hueso and Ralph Gay at CIC nanoGUNE, where nanolithography 
and FTIR were performed. This work has been supported in part by ERC (Advanced Grant 789104-eNANO), the Spanish MINECO (grant nos. MAT2017-88492-R, SEV2015-0522, PCIN-2015-155, and MAT2016-78293-C6-6-R), the Catalan CERCA Program, the Basque Government (grant no. IT1255-19), Fundació Privada Cellex, and the U.S. National Science Foundation CAREER Award (grant no. 1552461).

\section{REFERENCES}

(1) Anker, J. N.; Hall, W. P.; Lyandres, O.; Shah, N. C.; Zhao, J.; Van Duyne, R. P. Biosensing with Plasmonic Nanosensors. Nat. Mater. 2008, 7, 442-453.

(2) Rodrigo, D.; Limaj, O.; Janner, D.; Etezadi, D.; García de Abajo, F. J.; Pruneri, V.; Altug, H. Mid-Infrared Plasmonic Biosensing with Graphene. Science 2015, 349, 165-168.

(3) Mak, K. F.; Shan, J. Photonics and Optoelectronics of 2D Semiconductor Transition Metal Dichalcogenides. Nat. Photonics 2016, 10, 216-226.

(4) Danckwerts, M.; Novotny, L. Optical Frequency Mixing at Coupled Gold Nanoparticles. Phys. Rev. Lett. 2007, 98, 026104.

(5) Smirnova, D.; Kivshar, Y. S. Multipolar Nonlinear Nanophotonics. Optica 2016, 3, 1241-1255.

(6) Chang, D. E.; Sörensen, A. S.; Hemmer, P. R.; Lukin, M. D. Quantum Optics with Surface Plasmons. Phys. Rev. Lett. 2006, 97, 053002.

(7) Fakonas, J. S.; Lee, H.; Kelaita, Y. A.; Atwater, H. A. TwoPlasmon Quantum Interference. Nat. Photonics 2014, 8, 317-320.

(8) Zhou, C.; Duan, X.; Liu, N. A Plasmonic Nanorod that Walks on DNA Origami. Nat. Commun. 2015, 6, 8102.

(9) Li, K. R.; Stockman, M. I.; Bergman, D. J. Self-Similar Chain of Metal Nanospheres as an Efficient Nanolens. Phys. Rev. Lett. 2003, 91, 227402.

(10) Nagpal, P.; Lindquist, N. C.; Oh, S.-H.; Norris, D. J. Ultrasmooth Patterned Metals for Plasmonics and Metamaterials. Science 2009, 325, 594-597.

(11) Fan, J. A.; Wu, C. H.; Bao, K.; Bao, J. M.; Bardhan, R.; Halas, N. J.; Manoharan, V. N.; Nordlander, P.; Shvets, G.; Capasso, F. SelfAssembled Plasmonic Nanoparticle Clusters. Science 2010, 328, 1135-1138.

(12) Keller, J. W.; Coplan, M. A. Electron Energy Loss Spectroscopy of $\mathrm{C}_{60}$. Chem. Phys. Lett. 1992, 193, 89-92.

(13) Stéphan, O.; Taverna, D.; Kociak, M.; Suenaga, K.; Henrard, L.; Colliex, C. Dielectric Response of Isolated Carbon Nanotubes Investigated by Spatially Resolved Electron Energy-Loss Spectroscopy: From Multiwalled to Single-Walled Nanotubes. Phys. Rev. B: Condens. Matter Mater. Phys. 2002, 66, 155422.

(14) Arenal, R.; Stéphan, O.; Kociak, M.; Taverna, D.; Loiseau, A.; Colliex, C. Electron Energy Loss Spectroscopy Measurement of the Optical Gaps on Individual Boron Nitride Single-Walled and Multiwalled Nanotubes. Phys. Rev. Lett. 2005, 95, 127601.

(15) Nagao, T.; Yaginuma, S.; Inaoka, T.; Sakurai, T. OneDimensional Plasmon in an Atomic-Scale Metal Wire. Phys. Rev. Lett. 2006, 97, 116802.

(16) Moresco, F.; Rocca, M.; Hildebrandt, T.; Henzler, M. Plasmon Confinement in Ultrathin Continuous Ag Films. Phys. Rev. Lett. 1999, 83, 2238-2241.

(17) Rugeramigabo, E. P.; Nagao, T.; Pfnür, H. Experimental Investigation of Two-Dimensional Plasmons in a $\mathrm{DySi}_{2}$ Monolayer on Si(111). Phys. Rev. B: Condens. Matter Mater. Phys. 2008, 78, 155402.

(18) Chung, H. V.; Kubber, C. J.; Han, G.; Rigamonti, S.; SanchezPortal, D.; Enders, D.; Pucci, A.; Nagao, T. Optical Detection of Plasmonic and Interband Excitations in 1-nm-Wide Indium Atomic Wires. Appl. Phys. Lett. 2010, 96, 243101.

(19) Rugeramigabo, E. P.; Tegenkamp, C.; Pfnür, H.; Inaoka, T.; Nagao, T. One-Dimensional Plasmons in Ultrathin Metallic Silicide Wires of Finite Width. Phys. Rev. B: Condens. Matter Mater. Phys. 2010, 81, 165407.
(20) Zhou, W.; Lee, J.; Nanda, J.; Pantelides, S. T.; Pennycook, S. J.; Idrobo, J. C. Atomically Localized Plasmon Enhancement in Monolayer Graphene. Nat. Nanotechnol. 2012, 7, 161-165.

(21) Guler, U.; Boltasseva, A.; Shalaev, V. M. Refractory Plasmonics. Science 2014, 344, 263-264.

(22) Shah, D.; Reddy, H.; Kinsey, N.; Shalaev, V. M.; Boltasseva, A. Optical Properties of Plasmonic Ultrathin TiN Films. Adv. Mater. 2017, 5, 2816-2824.

(23) Boltasseva, A.; Shalaev, V. M. Transdimensional Photonics. ACS Photonics 2019, 6, 1-3.

(24) Fei, Z.; Rodin, A. S.; Andreev, G. O.; Bao, W.; McLeod, A. S.; Wagner, M.; Zhang, L. M.; Zhao, Z.; Thiemens, M.; Dominguez, G.; Fogler, M. M.; Neto, A. H. C.; Lau, C. N.; Keilmann, F.; Basov, D. N. Gate-Tuning of Graphene Plasmons Revealed by Infrared NanoImaging. Nature 2012, 487, 82-85.

(25) Chen, J.; Badioli, M.; Alonso-González, P.; Thongrattanasiri, S.; Huth, F.; Osmond, J.; Spasenović, M.; Centeno, A.; Pesquera, A.; Godignon, P.; Zurutuza Elorza, A.; Camara, N.; de Abajo, F. J. G.; Hillenbrand, R.; Koppens, F. H. L. Optical Nano-Imaging of GateTunable Graphene Plasmons. Nature 2012, 487, 77-81.

(26) Ni, G. X.; McLeod, A. S.; Sun, Z.; Wang, L.; Xiong, L.; Post, K. W.; Sunku, S. S.; Jiang, B.-Y.; Hone, J.; Dean, C. R.; Fogler, M. M.; Basov, D. N. Fundamental Limits to Graphene Plasmonics. Nature 2018, 557, 530-533.

(27) Basov, D. N.; Fogler, M. M.; García de Abajo, F. J. Polaritons in van der Waals Materials. Science 2016, 354, aag1992.

(28) Alcaraz Iranzo, D.; Nanot, S.; Dias, E. J. C.; Epstein, I.; Peng, C.; Efetov, D. K.; Lundeberg, M. B.; Parret, R.; Osmond, J.; Hong, J.Y.; Kong, J.; Englund, D. R.; Peres, N. M. R.; Koppens, F. H. L. Probing the Ultimate Plasmon Confinement Limits with a van der Waals Heterostructure. Science 2018, 360, 291-295.

(29) Di Pietro, P.; Ortolani, M.; Limaj, O.; Di Gaspare, A.; Giliberti, V.; Giorgianni, F.; Brahlek, M.; Bansal, N.; Koirala, N.; Oh, S.; Calvani, P.; Lupi, S. Observation of Dirac Plasmons in a Topological Insulator. Nat. Nanotechnol. 2013, 8, 556-560.

(30) Huber, M. A.; Mooshammer, F.; Plankl, M.; Viti, L.; Sandner, F.; Kastner, L. Z.; Frank, T.; Fabian, J.; Vitiello, M. S.; Cocker, T. L.; Huber, R. Femtosecond Photo-Switching of Interface Polaritons in Black Phosphorus Heterostructures. Nat. Nanotechnol. 2017, 12, 207-212.

(31) Manjavacas, A.; Marchesin, F.; Thongrattanasiri, S.; Koval, P.; Nordlander, P.; Sánchez-Portal, D.; García de Abajo, F. J. Tunable Molecular Plasmons in Polycyclic Aromatic Hydrocarbons. ACS Nano 2013, 7, 3635-3643.

(32) Lauchner, A.; Schlather, A.; Manjavacas, A.; Cui, Y.; McClain, M. J.; Stec, G. J.; García de Abajo, F. J.; Nordlander, P.; Halas, N. Nano Lett. 2015, 15, 6208-6214.

(33) Manjavacas, A.; García de Abajo, F. J. Tunable Plasmons in Atomically Thin Gold Nanodisks. Nat. Commun. 2014, 5, 3548.

(34) Yu, R.; Pruneri, V.; García de Abajo, F. J. Active Modulation of Visible Light with Graphene-Loaded Ultrathin Metal Plasmonic Antennas. Sci. Rep. 2016, 6, 32144.

(35) Kreibig, U.; Vollmer, M. Optical Properties of Metal Clusters; Springer-Verlag: Berlin, Germany, 1995.

(36) Scholl, J. A.; Koh, A. L.; Dionne, J. A. Quantum Plasmon Resonances of Individual Metallic Nanoparticles. Nature 2012, 483, 421-428.

(37) Maniyara, R. A.; Rodrigo, D.; Yu, R.; Canet-Ferrer, J.; Ghosh, D. S.; Yongsunthon, R.; Baker, D. E.; Rezikyan, A.; García de Abajo, F. J.; Pruneri, V. Tunable Plasmons in Ultrathin Metal Films. Nat. Photonics 2019, 13, 328-333.

(38) García de Abajo, F. J. Graphene Plasmonics: Challenges and Opportunities. ACS Photonics 2014, 1, 135-152.

(39) Neuhold, G.; Horn, K. Depopulation of the Ag(111) Surface State Assigned to Strain in Epitaxial Films. Phys. Rev. Lett. 1997, 78, 1327-1330.

(40) Chiang, T.-C. Photoemission Studies of Quantum Well States in Thin Films. Surf. Sci. Rep. 2000, 39, 181-235. 
(41) Speer, N. J.; Tang, S.-J.; Miller, T.; Chiang, T.-C. Coherent Electronic Fringe Structure in Incommensurate Silver-Silicon Quantum Wells. Science 2006, 314, 804-806.

(42) Schiller, F.; Abd El-Fattah, Z. M.; Schirone, S.; Lobo-Checa, J.; Urdanpilleta, M.; Ruiz-Oses, M.; Cordon, J.; Corso, M.; SánchezPortal, D.; Mugarza, A.; Ortega, J. E. Metallic Thin Films on Stepped Surfaces: Lateral Scattering of Quantum Well States. New J. Phys. 2014, 16, 123025.

(43) Bao, X.; Barth, J. V.; Lehmpfuhl, G.; Schuster, R.; Uchida, Y.; Schlögl, R.; Ertl, G. Oxygen-Induced Restructuring of Ag(111). Surf. Sci. 1993, 284, 14-22.

(44) Sanders, C. E.; Zhang, C.; Kellogg, G. L.; Shih, C.-K. Role of Thermal Processes in Dewetting of Epitaxial $\mathrm{Ag}(111)$ Film on Si(111). Surf. Sci. 2014, 630, 168-173.

(45) Namba, Y. Resistivity and Temperature Coefficient of Thin Metal Films with Rough Surface. Jpn. J. Appl. Phys. 1970, 9, 13261329.

(46) Luo, E. Z.; Heun, S.; Kennedy, M.; Wollschläger, J.; Henzler, M. Surface Roughness and Conductivity of Thin Ag Films. Phys. Rev. B: Condens. Matter Mater. Phys. 1994, 49, 4858-4865.

(47) Brandt, T.; Hövel, M.; Gompf, B.; Dressel, M. Temperatureand Frequency-Dependent Optical Properties of Ultrathin Au Films. Phys. Rev. B: Condens. Matter Mater. Phys. 2008, 78, 205409.

(48) Hövel, M.; Gompf, B.; Dressel, M. Dielectric Properties of Ultrathin Metal Films Around the Percolation Threshold. Phys. Rev. B: Condens. Matter Mater. Phys. 2010, 81, 035402.

(49) Daghero, D.; Paolucci, F.; Sola, A.; Tortello, M.; Ummarino, G. A.; Agosto, M.; Gonnelli, R. S.; Nair, J. R.; Gerbaldi, C. Large Conductance Modulation of Gold Thin Films by Huge Charge Injection via Electrochemical Gating. Phys. Rev. Lett. 2012, 108, 066807.

(50) Bondarev, I. V.; Shalaev, V. M. Universal Features of the Optical Properties of Ultrathin Plasmonic Films. Opt. Mater. Express 2017, 7, 3731-3740.

(51) Yu, R.; Liz-Marzán, L. M.; García de Abajo, F. J. Universal Analytical Modeling of Plasmonic Nanoparticles. Chem. Soc. Rev. 2017, 46, 6710-6724.

(52) Johnson, P. B.; Christy, R. W. Optical Constants of the Noble Metals. Phys. Rev. B 1972, 6, 4370-4379.

(53) McPeak, K. M.; Jayanti, S. V.; Kress, S. J. P.; Meyer, S.; Iotti, S.; Norris, D. J.; Rossinelli, A. Plasmonic Films Can Easily Be Better: Rules and Recipes. ACS Photonics 2015, 2, 326-333.

(54) He, J.-H.; Jiang, L.-Q.; Qiu, J.-L.; Chen, L.; Wu, K.-H. Growth of Atomically Flat Ultra-Thin $\mathrm{Ag}$ Films on $\mathrm{Si}(111)$ by Introducing a Growth of Atomically Flat Ultra-Thin Ag Films on $\mathrm{Si}(111)$ by Introducing a Square Root $3 \times$ Square Root 3-Ga Buffer Layer. Chin. Phys. Lett. 2014, 31, 128102.

(55) Starfelt, S.; Zhang, H. M.; Johansson, L. S. O. Quantum-Well States in Thin Ag Films Grown on the Ga/Si(111)-Square Root $3 \times$ Square Root 3 Surface. Phys. Rev. B: Condens. Matter Mater. Phys. 2018, 97, 195430.

(56) Schiller, F.; Cordón, J.; Rubio, D. V. A.; Ortega, J. E.; Vyalikh, D. Fermi Gap Stabilization of an Incommensurate Two-Dimensional Superstructure. Phys. Rev. Lett. 2005, 94, 016103.

(57) Miyazaki, M.; Hirayama, H. Initial Stage of Ag Growth on Bi/ $\mathrm{Ag}(111)$ Square Root $3 \times$ Square Root 3 Surfaces. Surf. Sci. 2008, 602, 276-282.

(58) Smits, F. M. Measurement of Sheet Resistivities with the FourPoint Probe. Bell Syst. Tech. J. 1958, 37, 711-718.

(59) Ashcroft, N. W.; Mermin, N. D. Solid State Physics; Harcourt College Publishers: Philadelphia, PA, 1976.

(60) Dias, E. J. C.; García de Abajo, F. J. Fundamental Limits to the Coupling between Light and 2D Polaritons. ACS Nano 2019, 13, 5184-5197.

(61) Yu, R.; Cox, J. D.; Saavedra, J. R. M.; García de Abajo, F. J. Analytical Modeling of Graphene Plasmons. ACS Photonics 2017, 4, 3106-3114.

(62) Aspnes, D. E.; Studna, A. A. Dielectric Functions and Optical Parameters of $\mathrm{Si}, \mathrm{Ge}, \mathrm{GaP}, \mathrm{GaAs}, \mathrm{GaSb}$, InP, InAs, and InSb from 1.5 to $6.0 \mathrm{eV}$. Phys. Rev. B: Condens. Matter Mater. Phys. 1983, 27, 9851009. 